POS $\quad$ PROCEEDINGS

\title{
Traces of the deconfined phase transition
}

\author{
Elena Bratkovskaya* \\ GSI Helmholtzzentrum für Schwerionenforschung GmbH Planckstrasse 1, 64291 Darmstadt, \\ Germany \\ E-mail: e.bratkovskaya@gsi.de
}

\section{Hamza Berrehrah}

Frankfurt Institute for Advanced Studies, Johann Wolfgang Goethe Universität, Ruth-Moufang-Strasse 1, 60438 Frankfurt am Main, Germany

E-mail: berrehrahefias.uni-frankfurt.de

\section{Wolfgang Cassing}

Institut für Theoretische Physik, Universität Giessen, 35392 Giessen, Germany

E-mail: wolfgang.cassing@theo.physik.uni-giessen.de

\section{Thorsten Steinert}

Institut für Theoretische Physik, Universität Giessen, 35392 Giessen, Germany

E-mail: thorsten.steinertatheo.physik.uni-giessen.de

\section{Taesoo Song}

Institut für Theoretische Physik, Universität Giessen, 35392 Giessen, Germany

E-mail: taesoo.song@theo.physik.uni-giessen.de

We address the dynamics of the partonic degrees of freedom in the deconfined phase on a microscopic basis. We report about results from an extended dynamical quasiparticle model (DQPM*) in which the effective parton propagators have a complex selfenergy that depends on the temperature $T$ of the medium as well as on the chemical potential $\mu_{q}$ and the parton three-momentum $\vec{p}$ with respect to the medium at rest. It is demonstrated that this approach allows for a good description of QCD thermodynamics with respect to the entropy density, pressure etc. above the critical temperature $T_{c} \approx 158 \mathrm{MeV}$ at $\mu_{q}=0$. Furthermore, the quark susceptibility $\chi_{q}$ and the quark number density $n_{q}$ are found to be reproduced simultaneously at zero and finite quark chemical potential. The shear and bulk viscosities $\eta, \zeta$, and the electric conductivity $\sigma_{e}$ from the DQPM* also turn out in close agreement with lattice results for $\mu_{q}=0$. The DQPM*, furthermore, allows to evaluate the momentum $p, T$ and $\mu_{q}$ dependencies of the partonic degrees of freedom also for larger $\mu_{q}$ which are mandatory for transport studies of heavy-ion collisions in the regime $5 \mathrm{GeV}$ $<\sqrt{s_{N N}}<50 \mathrm{GeV}$.

Critical Point and Onset of Deconfinement

7-11 August, 2017

The Wang Center, Stony Brook University, Stony Brook, NY

\footnotetext{
${ }^{*}$ Speaker.
} 


\section{Introduction}

The thermodynamic properties of the quark-gluon plasma (QGP)-as produced in relativistic heavy-ion collisions-are rather well determined within lattice QCD (IQCD) calculations at vanishing quark chemical potential $\mu_{q}[1,2]$. Whereas the results from different collaborations in the past have led to different equations of state (EoS) of partonic matter even at $\mu_{q}=0$ the present status can be considered as a consensus (within error bars). Nevertheless, the physical interpretation of the lattice 'data' remains a challenge since the EoS as well as transport coefficients from 1QCD indicate that the partonic system cannot be viewed as a weakly interacting medium of quark, antiquarks, and gluons. This holds especially true for temperatures close to the critical temperature $T_{c}$ where the entropy density $s(T)$ (and pressure $P(T)$ ) differ substantially from the Stefan Boltzmann limit. The 1QCD results on the EoS can conveniently be interpreted within quasiparticle models with massive partons $[3,4,5,6]$ that are fitted to the equation of state (EoS) from $1 \mathrm{QCD}$ and also allow for extrapolations to finite $\mu_{q}$, although with some ambiguities. However, in these effective models the spectral function of the degrees of freedom is taken as a $\delta$ - function (on-shell limit) which implies that these partons in principle are non-interacting. An extension of the simple quasiparticle model has been proposed in Refs. [7, 8, 9] where a finite width of the partonic spectral functions is introduced, which corresponds to the interaction rate of the parton in the medium at finite temperature $T$ and chemical potential $\mu_{q}$. The latter can be directly employed for the calculation of transport coefficients such as shear and bulk viscosities of the partonic medium in the relaxation time approximation [10] and be compared to corresponding correlators from IQCD. An interpretation in terms of quasiparticles, however, is constraint to effective propagators with a spectral width that is substantially smaller than the dynamical pole mass.

Furthermore, at non-zero quark chemical potential $\mu_{q} \neq 0$, the primary quantities of interest are the "pressure difference $\Delta P$ ", the quark number density $n_{B}$ and quark susceptibility $\chi_{q}$ since these quantities are available from IQCD [11,12]. The quark number susceptibilities are additional quantities to further quantify the properties of the partonic degrees of freedom (d.o.f.) especially in the vicinity of the QCD phase transition or crossover. It turns out that the standard quasiparticle models, that fit the partonic EoS, severely underestimate the quark susceptibilities. Nevertheless, the challenge of describing simultaneously both the 1QCD pressure and quark susceptibilities as well as transport coefficients is out of reach in these models [13]. Especially the quark susceptibilities are very sensitive to the quark masses used as inputs and solely determined by the quark degrees of freedom. On the other hand both light quark and gluon masses contribute to thermodynamic quantities like the entropy density $s$ and pressure $P$. Therefore, reconciling all observables from IQCD within a single effective model is a challenge.

In this contribution we will consider the QGP as a dynamical quasi-particle medium of massive off-shell particles with partonic propagators incorporating complex selfenergies which explicitly depend on the three-momentum $\mathbf{p}$ with respect to the partonic matter at rest in order to match perturbative QCD (pQCD) at high momenta. We will show that within the extended dynamical quasiparticle model - denoted by DQPM* - we reproduce the 1QCD equation of state at finite temperature $T$ and chemical potential $\mu_{q}$. Moreover, we simultaneously describe the quark number density and susceptibility $\chi_{q}$ from $1 Q C D$. In the same approach, we also compute the shear and bulk viscosities ( $\eta$ and $\zeta$ ) of the QGP at finite temperature $T$ and chemical potential $\mu_{q}$ in order to 
probe some transport properties of the partonic medium in comparison to lQCD.

\section{Parton properties in the DQPM*}

In the $\mathrm{DQPM}^{*}$ the entropy density $s(T)$, the pressure $P(T)$ and energy density $\varepsilon(T)$ are calculated in a straight forward manner by starting with the entropy density in the quasiparticle limit from Baym [9, 14],

$$
\begin{aligned}
& s^{d q p}=-d_{g} \int \frac{d \omega}{2 \pi} \frac{d^{3} p}{(2 \pi)^{3}} \frac{\partial f_{B}}{\partial T}\left(\mathfrak{I} \ln \left(-\Delta^{-1}\right)+\mathfrak{I} \Pi \Re \Delta\right) \\
& -d_{q} \int \frac{d \omega}{2 \pi} \frac{d^{3} p}{(2 \pi)^{3}} \frac{\partial f_{F}\left(\left(\omega-\mu_{q}\right) / T\right)}{\partial T}\left(\mathfrak{I n}\left(-S_{q}^{-1}\right)+\mathfrak{I} \Sigma_{q} \Re S_{q}\right) \\
& -d_{\bar{q}} \int \frac{d \omega}{2 \pi} \frac{d^{3} p}{(2 \pi)^{3}} \frac{\partial f_{F}\left(\left(\omega+\mu_{q}\right) / T\right)}{\partial T}\left(\mathfrak{I n}\left(-S_{\bar{q}}^{-1}\right)+\mathfrak{I} \Sigma_{\bar{q}} \mathfrak{R} S_{\bar{q}}\right),
\end{aligned}
$$

where $f_{B}(\omega / T)=(\exp (\omega / T)-1)^{-1}$ and $f_{F}\left(\left(\omega-\mu_{q}\right) / T\right)=\left(\exp \left(\left(\omega-\mu_{q}\right) / T\right)+1\right)^{-1}$ denote the Bose and Fermi distribution functions, respectively, while $\Delta=\left(P^{2}-\Pi\right)^{-1}, S_{q}=\left(P^{2}-\Sigma_{q}\right)^{-1}$ and $S_{\bar{q}}=\left(P^{2}-\Sigma_{\bar{q}}\right)^{-1}$ stand for the full (scalar) quasiparticle propagators of gluons $g$, quarks $q$ and antiquarks $\bar{q}$. In Eq. (2.1) $\Pi$ and $\Sigma=\Sigma_{q} \approx \Sigma_{\bar{q}}$ denote the (retarded) quasiparticle selfenergies. In principle, $\Pi$ as well as $\Delta$ are Lorentz tensors and should be evaluated in a nonperturbative framework. The $\mathrm{DQPM}^{*}$ treats these degrees of freedom as independent scalar fields with scalar selfenergies which are assumed to be identical for quarks and antiquarks. Note that one has to treat quarks and antiquarks separately in Eq. (2.1) as their abundance differs at finite quark chemical potential $\mu_{q}$. In Eq. (2.1) the degeneracy for gluons is $d_{g}=2\left(N_{c}^{2}-1\right)=16$ while $d_{q}=d_{\bar{q}}=2 N_{c} N_{f}=18$ is the degeneracy for quarks and antiquarks with three flavors. In practice one also has to differentiate between $(u, d)$ and $s$ quarks due to their mass difference.

As a next step one writes the complex selfenergies as $\Pi(\mathbf{q})=M_{g}^{2}(\mathbf{q})-2 i \omega \gamma_{g}(\mathbf{q})$ and $\Sigma_{q}(\mathbf{q})=$ $M_{q}(\mathbf{q})^{2}-2 i \omega \gamma_{q}(\mathbf{q})$ with a mass (squared) term $M^{2}$ and an interaction width $\gamma$, i.e. the retarded propagators $\left(\Delta, S_{q}\right)$ read,

$$
G_{R}(\omega, \mathbf{q})=\left(\omega^{2}-\mathbf{q}^{2}-M^{2}(\mathbf{q})+2 i \gamma(\mathbf{q}) \omega\right)^{-1},
$$

and are analytic in the upper half plane in the energy $\omega$ since the poles of $G_{R}$ are located in the lower half plane. The imaginary part of $G_{R}$ (2.2) then gives the spectral function of the degree of freedom (except for a factor $1 / \pi$ ). In the standard DQPM [9] the masses had been fixed in the spirit of the hard thermal loop (HTL) approach with the masses being proportional to an effective coupling $g\left(T / T_{c}\right)$ which has been enhanced in the infrared. In the $\mathrm{DQPM}^{*}$ the selfenergies depend additionally on the three-momentum $\mathbf{p}$ with respect to the medium at rest, while the dependence on the temperature $T / T_{c}$ and chemical potential $\mu_{q}$ are very similar to the standard DQPM [9].

\subsection{Masses, widths and spectral functions of partons in DQPM*}

The functional forms for the parton masses and widths at finite temperature $T$, quark chemical potential $\mu_{q}$ and momentum $p=|\mathbf{p}|$ are assumed to be given by

$$
M_{g}\left(T, \mu_{q}, p\right)=\left(\frac{3}{2}\right)\left[\frac{g^{2}\left(T^{\star} / T_{c}\left(\mu_{q}\right)\right)}{6}\left[\left(N_{c}+\frac{N_{f}}{2}\right) T^{2}+\frac{N_{c}}{2} \sum_{q} \frac{\mu_{q}^{2}}{\pi^{2}}\right]\right]^{1 / 2} \times h\left(\Lambda_{g}, p\right)+m_{\chi g},
$$




$$
\begin{aligned}
& M_{q, \bar{q}}\left(T, \mu_{q}, p\right)=\left[\frac{N_{c}^{2}-1}{8 N_{c}} g^{2}\left(T^{\star} / T_{c}\left(\mu_{q}\right)\right)\left[T^{2}+\frac{\mu_{q}^{2}}{\pi^{2}}\right]\right]^{1 / 2} \times h\left(\Lambda_{q}, p\right)+m_{\chi q}, \\
& \gamma_{g}\left(T, \mu_{q}, p\right)=N_{c} \frac{g^{2}\left(T^{\star} / T_{c}\left(\mu_{q}\right)\right)}{8 \pi} T \ln \left(\frac{2 c}{g^{2}\left(T^{\star} / T_{c}\left(\mu_{q}\right)\right)}+1.1\right)^{3 / 4} \times h\left(\Lambda_{g}, p\right), \\
& \gamma_{q, \bar{q}}\left(T, \mu_{q}, p\right)=\frac{N_{c}^{2}-1}{2 N_{c}} \frac{g^{2}\left(T^{\star} / T_{c}\left(\mu_{q}\right)\right)}{8 \pi} T \ln \left(\frac{2 c}{g^{2}\left(T^{\star} / T_{c}\left(\mu_{q}\right)\right)}+1.1\right)^{3 / 4} \times h\left(\Lambda_{q}, p\right),
\end{aligned}
$$

with the momentum-dependent function

$$
h(\Lambda, p)=\left[\frac{1}{1+\Lambda\left(T_{c}\left(\mu_{q}\right) / T^{\star}\right) p^{2}}\right]^{1 / 2},
$$

where $T^{\star 2}=T^{2}+\mu_{q}^{2} / \pi^{2}$ is the effective temperature used to extend the $\mathrm{DQPM}^{*}$ to finite $\mu_{q}$, while $\Lambda_{g}\left(T_{c}\left(\mu_{q}\right) / T^{\star}\right)=5\left(T_{c}\left(\mu_{q}\right) / T^{\star}\right)^{2} \mathrm{GeV}^{-2}$ and $\Lambda_{q}\left(T_{c}\left(\mu_{q}\right) / T^{\star}\right)=12\left(T_{c}\left(\mu_{q}\right) / T^{\star}\right)^{2} \mathrm{GeV}^{-2}$. Furthermore, $m_{\chi g} \approx 0.5 \mathrm{GeV}$ is the gluon condensate and $m_{\chi q}$ is the light-quark chiral mass ( $m_{\chi q}=0.003$ $\mathrm{GeV}$ for $u, d$ quarks and $m_{\chi q}=0.06 \mathrm{GeV}$ for $s$ quarks). Since the effective quark masses in the QGP are large compared to the chiral masses the latter can in practice be neglected. In Eq. (2.3) $m_{\chi g}\left(m_{\chi q}\right)$ gives the finite gluon (light quark) mass in the limit $p \rightarrow 0$ and $T=0$ or for $p \rightarrow \infty$. As mentioned above the quasiparticle masses and widths (2.3) are parametrized following hard thermal loop (HTL) functional dependencies at finite temperature as in the default DQPM [9] in order to follow the correct high temperature limit. The essentially new elements in (2.3) are the multiplicative factors $h(\Lambda, p)$ (2.4) specifying the momentum dependence of the masses and widths with additional parameters $\Lambda_{g}$ and $\Lambda_{q}$ and the additive terms $m_{\chi g}$ and $m_{\chi q}$. The momentum-dependent factor $h(\Lambda, p)$ in the masses (2.3) is motivated by Dyson-Schwinger studies in the vacuum [15] and yields the limit of $\mathrm{pQCD}$ for $p \rightarrow \infty$.

The effective gluon and quark masses are a function of $T^{\star}$ at finite $\mu_{q}$. Here we consider three light flavors $(q=u, d, s)$ and assume all chemical potentials to be equal $\left(\mu_{u}=\mu_{d}=\mu_{s}=\mu_{q}\right)$. Note that alternative settings are also possible to comply with strangeness neutrality in heavy-ion collisions. The coupling (squared) $g^{2}$ in Eq. (2.3) is the effective running coupling given as a function of $T / T_{c}$ at $\mu_{q}=0$. A straight forward extension of the DQPM* to finite $\mu_{q}$ is to consider the coupling as a function of $T^{\star} / T_{c}\left(\mu_{q}\right)$ with a $\mu_{q}$-dependent critical temperature $T_{c}\left(\mu_{q}\right)$,

$$
T_{c}\left(\mu_{q}\right)=T_{c}\left(\mu_{q}=0\right) \sqrt{1-\alpha \mu_{q}^{2}} \approx T_{c}\left(\mu_{q}=0\right)\left(1-\alpha / 2 \mu_{q}^{2}+\ldots\right)
$$

with $\alpha \approx 8.79 \mathrm{GeV}^{-2}$. We recall that the expression of $T_{c}\left(\mu_{q}\right)$ in Eq. (2.5) is obtained by requiring a constant energy density $\varepsilon$ for the system at $T=T_{c}\left(\mu_{q}\right)$ where $\varepsilon$ at $T_{c}\left(\mu_{q}=0\right) \approx 0.158 \mathrm{GeV}$ is fixed by a lattice QCD calculation at $\mu_{q}=0$. The coefficient in front of the $\mu_{q}^{2}$-dependent part can be compared to 1QCD calculations at finite (but small) $\mu_{B}$ which gives [16]

$$
T_{c}\left(\mu_{B}\right)=T_{c}\left(\mu_{B}=0\right)\left(1-\kappa\left(\frac{\mu_{B}}{T_{c}\left(\mu_{B}=0\right)}\right)^{2}+\ldots\right)
$$

with $\kappa=0.013(2)$. Rewriting (2.5) in the form (2.6) and using $\mu_{B} \approx 3 \mu_{q}$ we get $\kappa_{D Q P M} \approx 0.0122$ which compares well with the $1 \mathrm{QCD}$ result. 
Using the pole masses and widths (2.3), the spectral functions for the partonic degrees of freedom are fully determined, i.e. the imaginary parts of the retarded propagators. The real part of the retarded propagators then follows from dispersion relations or directly from Eq. (2). Since the retarded propagators show no poles in the upper complex half plane in the energy $\omega$ the model propagators obey micro-causality. The imaginary parts are of Lorentzian form and provide the spectral functions $[8,9]$,

$$
\rho_{i}(\omega, \mathbf{p})=\frac{\gamma_{i}(\mathbf{p})}{\tilde{E}_{i}(\mathbf{p})}\left(\frac{1}{\left(\omega-\tilde{E}_{i}(\mathbf{p})\right)^{2}+\gamma_{i}^{2}(\mathbf{p})}-\frac{1}{\left(\omega+\tilde{E}_{i}(\mathbf{p})\right)^{2}+\gamma_{i}^{2}(\mathbf{p})}\right)
$$

with $\tilde{E}_{i}^{2}(\mathbf{p})=\mathbf{p}^{2}+M_{i}^{2}(\mathbf{p})-\gamma_{i}^{2}(\mathbf{p})$ for $i \in[g, q, \bar{q}]$. These spectral functions (2.7) are antisymmetric in $\omega$ and normalized as

$$
\int_{-\infty}^{+\infty} \frac{d \omega}{2 \pi} \omega \rho_{i}(\omega, \mathbf{p})=\int_{0}^{+\infty} \frac{d \omega}{2 \pi} 2 \omega \rho_{i}(\omega, \mathbf{p})=1,
$$

where $M_{i}\left(T, \mu_{q}, \mathbf{p}\right), \gamma_{i}\left(T, \mu_{q}, \mathbf{p}\right)$ are the particle pole mass and width at finite three momentum $\mathbf{p}$, temperature $T$ and chemical potential $\mu_{q}$, respectively.

In contrast to previous DQPM studies we employ here a new method for the determination of the effective coupling which is more flexible and can be directly extracted from the 1QCD data on the entropy density $s(T)$. For details we refer the reader to the review [17] and focus on the actual results. Figs. 1 (a)-(b) show the gluon and light quark masses and widths, respectively, at finite temperature and chemical potential for a momentum $p=1 \mathrm{GeV} / \mathrm{c}$. Furthermore, Fig. 1 (c) shows the gluon and light quark masses as a function of momentum (squared) $p^{2}$ at finite temperature $T=2 T_{c}$ and different $\mu_{q}$. Note that for $p=0$ we obtain higher values of the gluon and light quark masses (as a function of $T$ and $\mu_{q}$ ) since for finite momenta the masses decrease (at a given temperature and chemical potential), especially for the light quarks as seen in Fig 1 (c). The extension $T / T_{c} \rightarrow T^{\star} / T_{c}\left(\mu_{q}\right)$ for finite $\mu_{q}$ in the functional form for the strong coupling leads to lower values for the parton masses and widths at finite $\mu_{q}$ as compared to $\mu_{q}=0$ near $T_{c}\left(\mu_{q}\right)$.
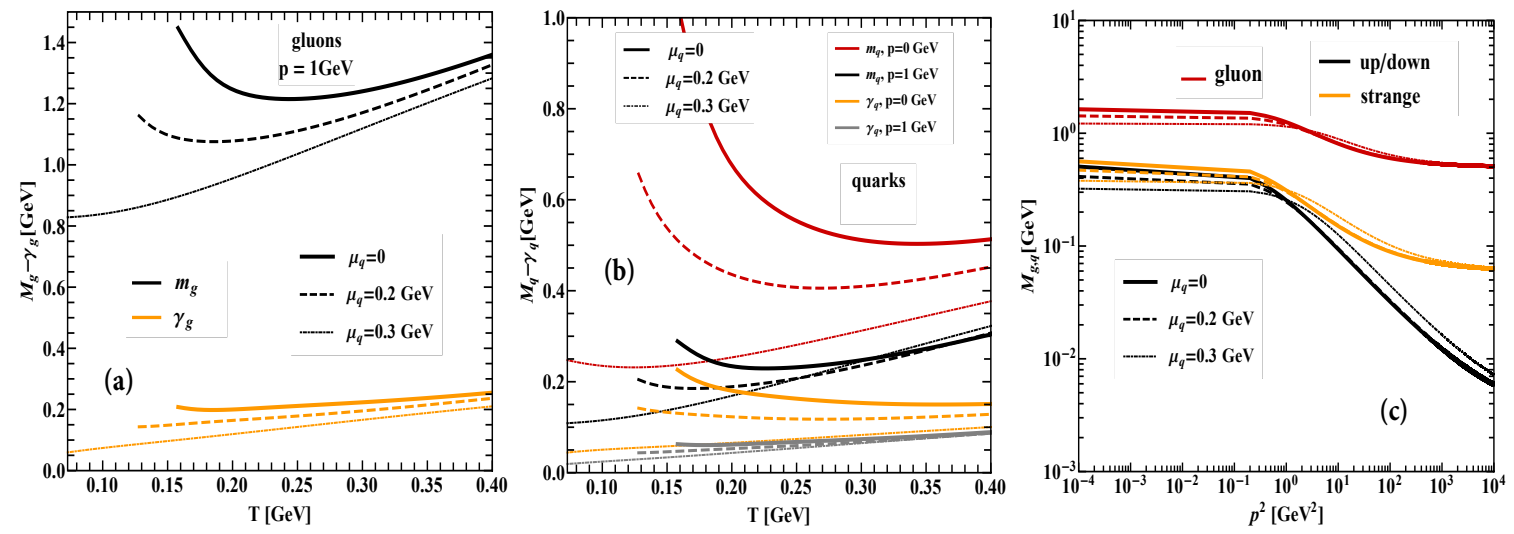

Figure 1: The DQPM* gluon (a) and light quark (b) masses and widths given by (2.3) for different quark chemical potentials as a function of the temperature T. (c) Gluon and light quark masses as a function of the momentum squared for $T=2 T_{c}$ and $\mu_{q}=0,0.2,0.3 \mathrm{GeV}$. The figures are taken from Ref. [18]. 


\subsection{Thermodynamics of the QGP from DQPM*}

The expressions for the equation of state (energy density $\varepsilon$, entropy density $s$ and pressure $P$ ) of strongly interacting matter have been given for finite temperature and chemical potential in Ref. [10] for on-shell partons and in Ref. [9] for the case of off-shell partons using the relations based on the stress-energy tensor $T^{\mu v}$. We recall that the approach for calculating the equation of state in the $\mathrm{DQPM}^{*}$ is based on thermodynamic relations (see below). The procedure is as follows: One starts from the evaluation of the entropy density $s$ from (2.1) employing the masses and widths obtained from the expressions in Section 2.1. Then using the thermodynamic relation $s=(\partial P / \partial T)_{\mu_{q}}$ (for a fixed quark chemical potential $\mu_{q}$ ) one obtains the pressure $P$ by integration of the entropy density $s$ over $T$ while the energy density $\varepsilon$ can be gained using the relation,

$$
\varepsilon\left(T, \mu_{B}\right)=T s\left(T, \mu_{B}\right)-P\left(T, \mu_{B}\right)+\mu_{B} n_{B}\left(T, \mu_{B}\right),
$$

where $n_{B}$ is the net baryon density.
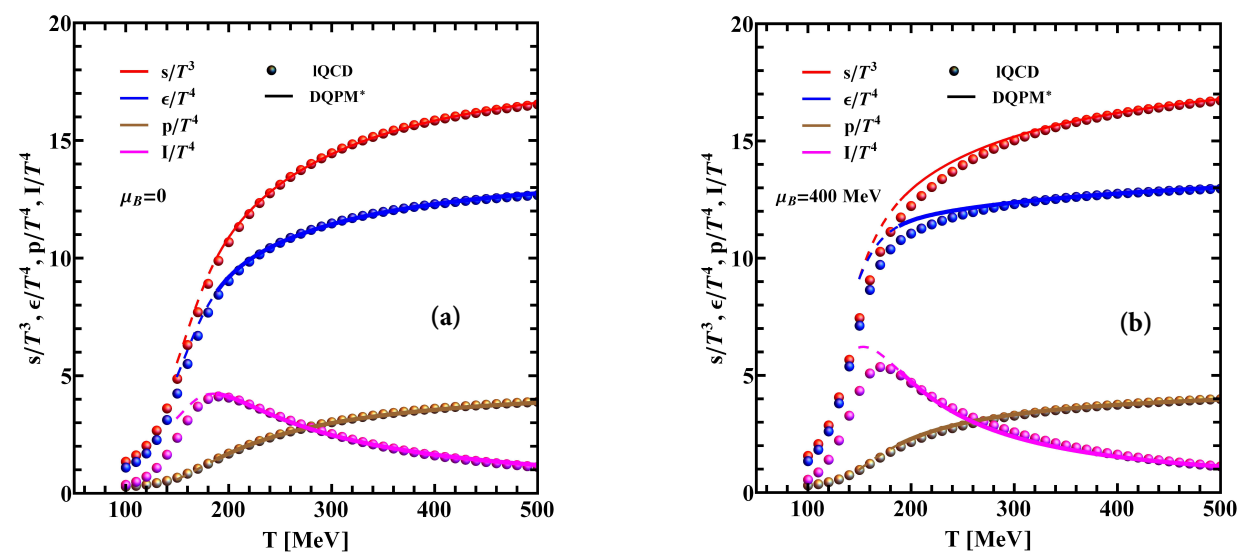

Figure 2: Scaled energy density $\varepsilon$, entropy density s, pressure $P$ and trace anomaly $(I=\varepsilon-3 P)$ as a function of temperature $T$ at $\mu_{B}=0\left(\right.$ a) and at $\mu_{B}=400 \mathrm{MeV}(b)$ from $D Q P M^{*}$ compared to lQCD data from Ref. [11]. The figures are taken from Ref. [18].

The energy density $\varepsilon$, entropy density $s$, pressure $P$ and the interaction measure $\left[I\left(T, \mu_{q}\right)=\right.$ $\left.\varepsilon\left(T, \mu_{q}\right)-3 P\left(T, \mu_{q}\right)\right]$-known in $1 \mathrm{QCD}$ as the trace anomaly- in the $\mathrm{DQPM}^{*}$ are shown in Fig. 2 (a), (b) as a function of temperature $T$ for two values of the baryon chemical potential $\mu_{B}=0$ and $\mu_{B}=400 \mathrm{MeV}$, respectively (where $\mu_{B}=3 \mu_{q}$ in our study). We, furthermore, compare our results with lattice calculations from Ref. [11] and notice that our results are in a very good agreement with the lattice data for $\mu_{B}=0$ (a) and in case of $\mu_{B}=400 \mathrm{MeV}$ (b) for temperatures larger than $1.2 T_{c}\left(\mu_{q}\right)$. In the latter case we observe (for temperatures just above $T_{c}(\mu)$ ) some deviations which are expected to result from additional hadronic degrees of freedom in the crossover region.

At finite baryon chemical potential, i.e. $\mu_{B}=400 \mathrm{MeV}$, the maximum of the trace anomaly is shifted towards lower temperatures. We notice also the proper scaling of our DQPM* description of QGP thermodynamics, when moving from zero to finite quark chemical potential (cf. Fig.2 (a) and (b)). 


\section{Quark number density and susceptibility from DQPM*}

\subsection{Baryon number density in the DQPM*}

The equation of state for vanishing chemical potential $\mu_{q}=0$ is defined fully by the entropy density $s$; for finite chemical potential $\mu_{q} \neq 0$ one has to include the particle density $n$. In the DQPM* the quark density $n^{d q p}$ in the quasiparticle limit is defined in analogy to the entropy density (2.1) as [19],

$$
\begin{aligned}
n^{d q p}= & -d_{q} \int \frac{d \omega}{2 \pi} \frac{d^{3} p}{(2 \pi)^{3}} \frac{\partial f_{F}\left(\left(\omega-\mu_{q}\right) / T\right)}{\partial \mu_{q}}\left(\mathfrak{I} \ln \left(-S_{q}^{-1}\right)+\mathfrak{I} \Sigma_{q} \mathfrak{R} S_{q}\right) \\
& -d_{\bar{q}} \int \frac{d \omega}{2 \pi} \frac{d^{3} p}{(2 \pi)^{3}} \frac{\partial f_{F}\left(\left(\omega+\mu_{q}\right) / T\right)}{\partial \mu_{q}}\left(\mathfrak{I} \ln \left(-S_{\bar{q}}^{-1}\right)+\mathfrak{I} \Sigma_{\bar{q}} \mathfrak{R} S_{\bar{q}}\right) .
\end{aligned}
$$

In case of the Lorentzian spectral function the density $n^{d q p}$ in Eq. (3.1) can be split into the following two terms $n_{q}^{(0)}$ and $\Delta n_{q}$ as:

$$
\begin{gathered}
n_{q}^{(0)}=d_{q} \int \frac{d^{3} p}{(2 \pi)^{3}} f_{q}^{(0)}-d_{\bar{q}} \int \frac{d^{3} p}{(2 \pi)^{3}} f_{\bar{q}}^{(0)}, \\
\Delta n_{q}=d_{q} \int \frac{d \omega}{(2 \pi)} \frac{d^{3} p}{(2 \pi)^{3}} \frac{\partial f_{q}\left(\left(\omega-\mu_{q}\right) / T\right)}{\partial \mu_{q}} \xi(\omega, p) \\
+d_{\bar{q}} \int \frac{d \omega}{(2 \pi)} \frac{d^{3} p}{(2 \pi)^{3}} \frac{\partial f_{\bar{q}}\left(\left(\omega+\mu_{q}\right) / T\right)}{\partial \mu_{q}} \xi(\omega, p),
\end{gathered}
$$

with

$$
\xi(\omega, p)=\left(2 \gamma \omega \frac{\omega^{2}-\mathbf{p}^{2}-M^{2}}{\left(\omega^{2}-\mathbf{p}^{2}-M^{2}\right)^{2}+4 \gamma^{2} \omega^{2}}-\arctan \left(\frac{2 \gamma \omega}{\omega^{2}-\mathbf{p}^{2}-M^{2}}\right)\right)
$$

where $f_{q}^{(0)}=\left(\exp \left(\left(\sqrt{p^{2}+M^{2}}-\mu_{q}\right) / T\right)+1\right)^{-1}, f_{\bar{q}}^{(0)}=\left(\exp \left(\left(\sqrt{p^{2}+M^{2}}+\mu_{q}\right) / T\right)+1\right)^{-1}$ denote again the Fermi distribution functions for the on-shell quark and anti-quark, with $M$ corresponding to the pole mass.

Finally, note that the quark number density (3.1) follows from the same potential as the entropy density [14] which ensures that it fulfills the thermodynamic relation $n=\left(\partial P / \partial \mu_{q}\right)_{T}$ (for fixed temperature $\mathrm{T})$. To be fully thermodynamically consistent the entropy and the particle density have to satisfy the Maxwell relation $(\partial n / \partial T)_{\mu_{q}}=\left(\partial s / \partial \mu_{q}\right)_{T}$. This provides further constraints on the effective coupling $g^{2}\left(T, \mu_{q}\right)$ at finite chemical potential which we neglect in the current approach. Nevertheless, it was checked that the violation of the latter Maxwell relation is generally small (up to $\mu_{B} \approx 500 \mathrm{MeV}$ ) and most pronounced around $T_{c}$. The baryon number density $n_{B}$, finally, is related to the quark number density by the simple relation $n_{B}=n^{d q p} / 3$.

\subsection{Susceptibilities in the DQPM*}

From the densities $n_{B}$ one may obtain other thermodynamic quantities like the pressure difference $\Delta P$ and the quark susceptibilities $\chi_{q}$, which can be confronted with lattice data for $N_{f}=2$ from Alton et al. [20, 21] and for $N_{f}=3$ from Borsanyi et al. [11]. We recall that the quark-number 
susceptibility measures the static response of the quark number density to an infinitesimal variation of the quark chemical potential. From Eqs. (3.2)-(3.4) we calculate $\Delta P$ and $\chi_{q}$ as

$$
\begin{gathered}
\Delta P\left(T, \mu_{B}\right) \equiv P\left(T, \mu_{B}\right)-P(T, 0)=\int_{0}^{\mu_{B}} n_{B} d \mu_{B} ; \\
\chi_{q}(T)=\left.\frac{\partial n_{q}}{\partial \mu_{q}}\right|_{\mu_{q}=0} ; \quad \chi_{q}\left(T, \mu_{q}\right)=9 \frac{\partial n_{B}}{\partial \mu_{B}} .
\end{gathered}
$$

Furthermore, for small $\mu_{q}$ a Taylor expansion of the pressure in $\mu_{q} / T$ can be performed which gives

$$
\frac{P\left(T, \mu_{q}\right)}{T^{4}}=\sum_{n=0}^{\infty} c_{n}(T)\left(\frac{\mu_{q}}{T}\right)^{n}, \quad c_{n}(T)=\left.\frac{1}{n !} \frac{\partial^{n}\left(P\left(T, \mu_{q}\right) / T^{4}\right)}{\partial\left(\mu_{q} / T\right)^{n}}\right|_{\mu_{q}=0},
$$

where $c_{n}(T)$ is vanishing for odd $n$ and $c_{0}(T)$ is given by $c_{0}(T)=P\left(T, \mu_{q}=0\right)$. As shown above the DQPM* compares well with lattice QCD results for $c_{0}(T)$. Since $\chi_{q}$ at finite $\mu_{q}$ is related to the pressure by

$$
\chi_{q}\left(T, \mu_{q}\right) / T^{2}=\partial^{2}\left(P / T^{4}\right) / \partial^{2}\left(\mu_{q} / T\right),
$$

one can define the susceptibility $\chi_{2}^{i j}$ at vanishing quark chemical potential as [11]

$$
\frac{P\left(T, \mu_{i}\right)}{T^{4}}=\frac{P(T, 0)}{T^{4}}+\frac{1}{2} \sum_{i, j} \frac{\mu_{i} \mu_{j}}{T^{2}} \chi_{2}^{i j}, \text { with } \chi_{2}^{i j}=\left.\frac{1}{T^{2}} \frac{\partial n_{j}\left(T, \mu_{i}\right)}{\partial \mu_{i}}\right|_{\mu_{i}=\mu_{j}=0},
$$

which in case of 3 flavors with $\mu_{u}=\mu_{d}=\mu_{s}$ becomes

$$
\chi_{2}(T)=\left.9 \frac{1}{T^{2}} \frac{\partial n_{q}\left(T, \mu_{q}\right)}{\partial \mu_{q}}\right|_{\mu_{q}=0}=9 \frac{\chi_{q}(T)}{T^{2}}
$$

\section{$3.3 n_{B}$ and $\chi_{q}:$ DQPM$^{*}$ vs IQCD}

Using the masses and widths (2.3) we calculate the baryon number density $n_{B}$ (3.2)-(3.3) and quark susceptibility $\chi_{2}$ including the finite width of the parton spectral functions. The results for $n_{B}$ and $\chi_{2}$ for $N_{f}=3$ are displayed in Fig. 3 (a) and (b), respectively. The comparison with the lattice data from Ref. [11] is rather good which is essentially due to an extra contribution arising from the momentum dependence of the $\mathrm{DQPM}^{*}$ quasiparticles masses and widths. Such a momentum dependence in $m_{q, \bar{q}, g}$ and $\gamma_{q, \bar{q}, g}$ decreases the 'thermal average' of light quark and gluon masses which improves the description of $1 \mathrm{QCD}$ results for the susceptibilities. For comparison we also show the result for $\chi_{q}$ from the conventional DQPM, i.e. with momentum independent masses, which substantially underestimates the lattice data. The small difference between IQCD and DQPM* for $n_{B}$ and $\chi_{2}$ close to $T_{C}$ is related to a possible excess of light quarks and antiquarks which should combine to hadrons in the crossover region. We recall that the $\mathrm{DQPM}^{*}$ describes only the QGP phase and deals with dynamical quarks and gluons solely. 
Finally, we emphasize the challenge to describe simultaneously the entropy $s$ and pressure $P$ on one side and $n_{B}$ and $\chi_{2}$ on the other side. Indeed, increasing the light quark mass and width helps to improve the description of $s$ and $P$ (for $\mu_{B}=400 \mathrm{MeV}$ ), but this leads to a considerable decrease in $n_{B}$ and $\chi_{2}$. In other words, lighter quarks are favorable to improve the agreement with 1QCD data on $n_{B}$ and $\chi_{2}$, however, this leads to an increase of $s$ and $P$, which can be only partially counterbalanced by an increasing gluon mass and width (which do not enter $n_{B}$ and $\chi_{2}$ ).
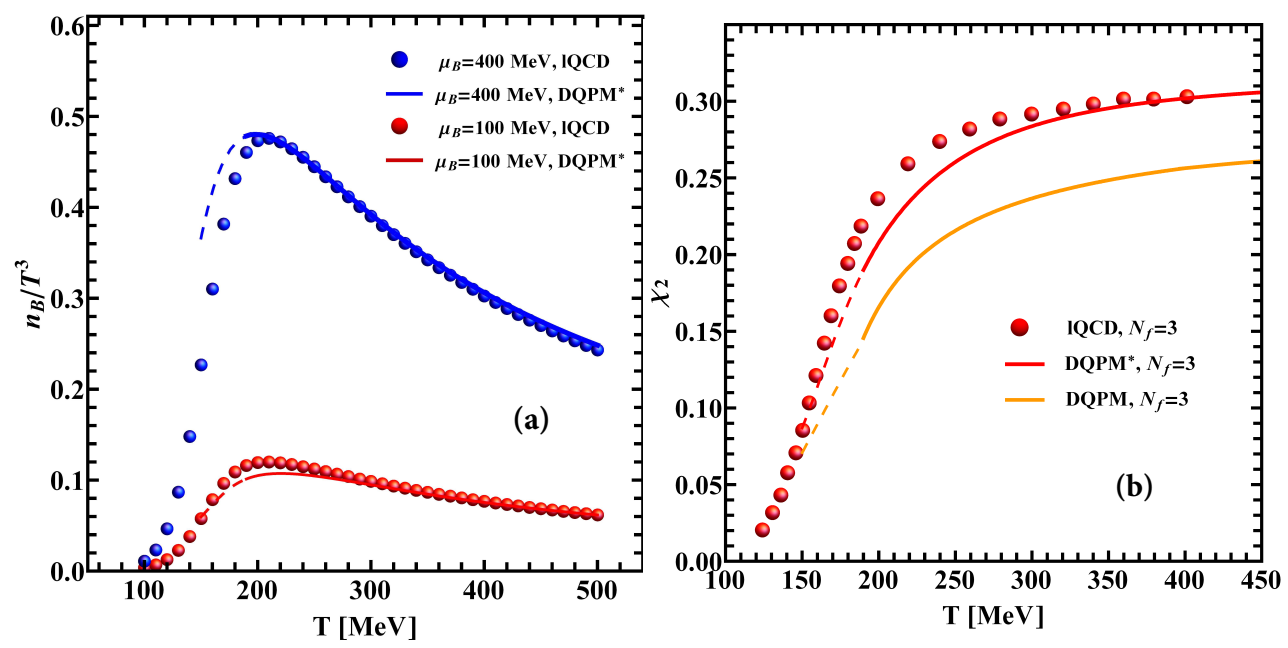

Figure 3: (a) The baryon number density $n_{B} / T^{3}$ from $D Q P M^{*}$ as compared to lattice data from Ref. [11] for $N_{f}=3$ for a quark chemical potential $\mu_{q}=0$. (b) The susceptibility $\chi_{2}$ from DQPM* as compared to lattice data from Ref.[11] for $N_{f}=3$ and $\mu_{q}=0$ using Eq. (3.9). The lower (orange) line gives the result from the conventional DQPM, i.e. with momentum independent masses. The figures are taken from Ref. [18].

\section{Transport properties of the hot QGP from DQPM*}

\subsection{Shear and bulk viscosities}

In this Section we report on the transport coefficients of the QGP using the relaxation time approximation (RTA). In the dilute gas approximation the relaxation time $\tau_{i}$ of the particle $i$ is obtained for on- or off-shell quasi-particles by means of the partonic scattering cross sections, where the $q q, q \bar{q}, q g$ and $g g$ elastic scattering processes as well as some inelastic processes involving chemical equilibration, such as $g g \rightarrow q \bar{q}$ are included in the computation of $\tau_{i}$ [22]. For the DQPM* approach we do not need the explicit cross sections since the inherent quasi-particle width $\gamma_{i}\left(T, \mu_{q}, p\right)$ directly provides the total interaction rate [9]. To this end we only have to evaluate the average of the momentum dependent widths $\gamma_{g}\left(T, \mu_{q}, p\right)$ and $\gamma_{q}\left(T, \mu_{q}, p\right)$ over the thermal distributions at fixed $T$ and $\mu_{q}$, i.e. $\bar{\gamma}_{g}\left(T, \mu_{q}\right)$ and $\bar{\gamma}_{q}\left(T, \mu_{q}\right)$.

The shear viscosity $\eta\left(T, \mu_{q}\right)$ is defined in the dilute gas approximation for the case of the DQPM* $^{*}$ off-shell particles by $[22,23]$

$$
\eta\left(T, \mu_{q}\right)=\frac{2}{15 T} d_{g} \int \frac{d^{3} p}{(2 \pi)^{3}} \int \frac{d \omega}{2 \pi} \omega \bar{\tau}_{g}\left(T, \mu_{q}\right) f_{g}(\omega / T) \times \rho_{g}(\omega, \mathbf{p}) \frac{\mathbf{p}^{4}}{\omega^{2}} \Theta\left(P^{2}\right)
$$




$$
\begin{aligned}
& +\frac{2}{15 T} \frac{d_{q}}{6} \int \frac{d^{3} p}{(2 \pi)^{3}} \int \frac{d \omega}{2 \pi} \omega\left[\sum_{q}^{u, d, s} \bar{\tau}_{q}\left(T, \mu_{q}\right) f_{q}\left(\left(\omega-\mu_{q}\right) / T\right) \rho_{q}(\omega, \mathbf{p})\right. \\
& \left.+\sum_{\bar{q}}^{\bar{u}, \bar{d}, \bar{s}} \bar{\tau}_{\bar{q}}\left(T, \mu_{q}\right) f_{\bar{q}}\left(\left(\omega+\mu_{q}\right) / T\right) \rho_{\bar{q}}(\omega, \mathbf{p})\right] \frac{\mathbf{p}^{4}}{\omega^{2}} \Theta\left(P^{2}\right),
\end{aligned}
$$

where $\mathbf{p}$ is the three-momentum and $P^{2}$ the invariant mass squared. The functions $\rho_{g}, \rho_{q}, \rho_{\bar{q}}$ stand for the gluon, quark and antiquark spectral functions, respectively, and $f_{q}\left(f_{\bar{q}}\right)$ stand for the equilibrium distribution functions for particle and antiparticle. The medium-dependent relaxation times $\bar{\tau}_{q, g}\left(T, \mu_{q}\right)$ in (4.1) are given in the $\mathrm{DQPM}^{*}$ by:

$$
\bar{\tau}_{q, g}\left(T, \mu_{q}\right)=\left(\bar{\gamma}_{q, g}\right)^{-1}\left(T, \mu_{q}\right)
$$

with:

$$
\begin{aligned}
& \bar{\gamma}_{q, g}\left(T, \mu_{q}\right)=\left\langle\gamma_{q, g}\left(T, \mu_{q}, p\right)\right\rangle_{p} \\
& =\left(n_{q, g}^{\mathrm{off}}\left(T, \mu_{q}\right)\right)^{-1} \times \int \frac{d^{3} p}{(2 \pi)^{3}} \frac{d \omega}{(2 \pi)} \omega \gamma_{q, g}\left(T, \mu_{q}, p\right) \rho_{f}(\omega) f_{q, g}\left(\omega, T, \mu_{q}\right) \Theta\left(P^{2}\right),
\end{aligned}
$$

where

$$
n_{f, g}^{\mathrm{off}}\left(T, \mu_{q}\right)=\int \frac{d^{3} p}{(2 \pi)^{3}} \frac{d \omega}{(2 \pi)} \omega \rho_{f}(\omega) f_{f, g}\left(\omega, T, \mu_{q}\right) \Theta\left(P^{2}\right)
$$

denotes the off-shell density of quarks, antiquarks or gluons. We note in passing that the shear viscosity $\eta$ can also be computed using the stress-energy tensor and the Green-Kubo formalism [24]. However, explicit comparisons of both methods in Ref. [24] have shown that the solutions are rather close. This holds especially for the case of the scattering of massive partons where the transport cross section is not very different from the total cross section as also pointed out in Ref. [25]. Furthermore, we mention that the definition of the shear viscosity $\eta$ is strictly valid only in the on-shell limit, however, can be employed also in the $\mathrm{DQPM}^{*}$ since the relaxation times $\bar{\tau}_{i}$ do not depend on the masses.

We show the DQPM* results for $\eta / s$, where $s$ is the DQPM* entropy density, in Fig.4 (a) as a function of the temperature. The (upper) orange solid line represents the case of the standard DQPM where the parton masses and widths are independent of momenta as calculated in Ref. [22]. The thick red solid line displays the result using Eqs. (4.1) and (4.2), where the parton masses and width are temperature, chemical potential and momentum dependent. Finally, the black solid line refers to the calculation of $\eta / s$ in Yang-Mills theory from the Kubo formula using an exact diagrammatic representation in terms of full propagators and vertices from Ref. [26].

Fig. 4 (a) shows that $\eta / s$ from DQPM* is in the range of the 1QCD data and significantly lower than the pQCD limit. As a function of temperature $\eta / s$ shows a minimum around $T_{c}$, similar to atomic and molecular systems [27] and then increases slowly for higher temperatures. This behavior is very much the same as in the standard DQPM (upper orange line) as shown in Ref. [24]. Therefore, the produced QGP shows features of a strongly interacting fluid unlike a weakly interacting parton gas as had been expected from perturbative QCD (pQCD). The minimum of $\eta / s$ at $T_{c}=158 \mathrm{MeV}$ is close to the lower bound of a perfect fluid with $\eta / s=1 /(4 \pi)$ [28] for infinitely 
coupled supersymmetric Yang-Mills gauge theory (based on the AdS/CFT duality conjecture). This suggests the "hot QCD matter" to be the "most perfect fluid" [27]. Furthermore, the ratio $\eta / s$ in

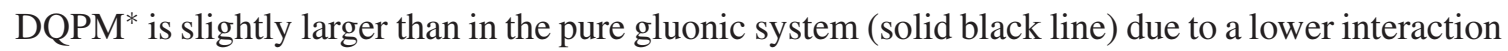
rate of quarks and antiquarks relative to gluons.

The explicit dependencies of $\eta / s$ on $T$ and $\mu_{q}$ are shown in Fig. 4 (b) where $\eta / s$ is seen to increase smoothly for finite but small $\mu_{q}$. We point out again that extrapolations to larger $\mu_{q}$ become increasingly uncertain.
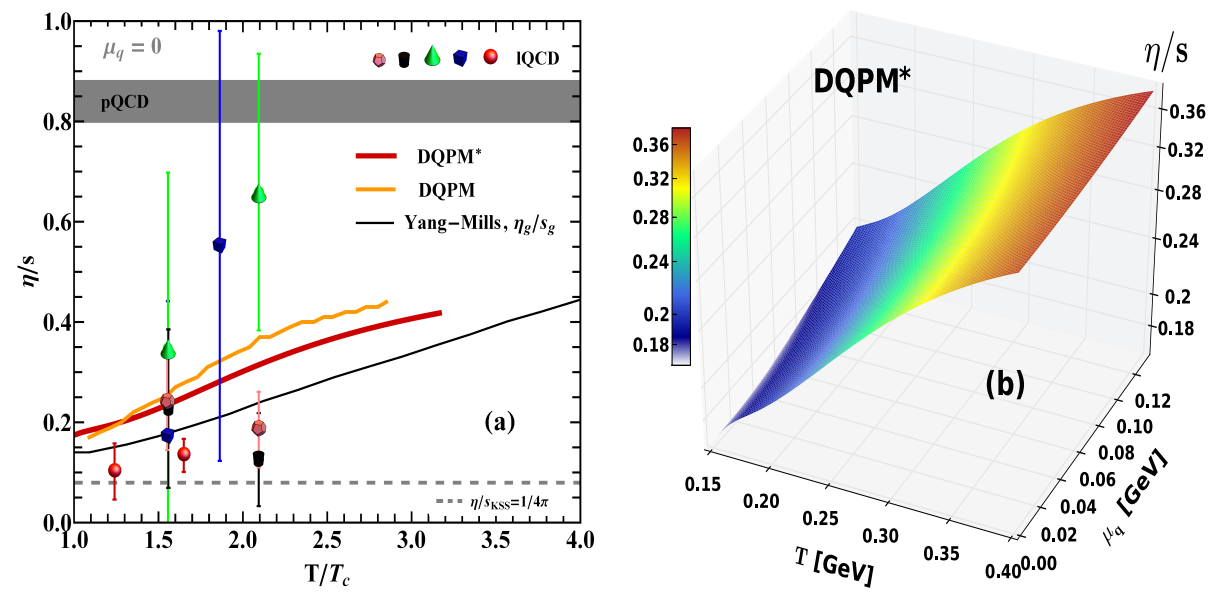

Figure 4: The shear viscosity to entropy density ratio $\eta / s$ from different models as a function of temperature $T$ for $\mu_{q}=0(a)$ and $\eta / s$ given by the DQPM* approach as a function of $\left(T, \mu_{q}\right)(b)$. The orange solid line in (a) results from the standard DQPM where the parton masses and widths are independent of momenta [22]. The thick red solid line shows the DQPM* result using Eqs.(4.1) and (4.2), where the parton masses and width are temperature, chemical potential and momentum dependent. The lattice QCD data for pure SU (3) gauge theory are taken from Ref. [33] (red spheres), from Ref. [29] (green pyramid and blue cubic), and from Ref. [30] (black cylinder and pink penthagone). The orange dashed line gives the Kovtun-SonStarinets lower bound [28] $(\eta / s)_{K S S}=1 /(4 \pi)$. Finally, the black solid line refers to the calculation of $\eta / s$ in Yang-Mills theory from Ref. [26]. The figures are taken from Ref. [18].

The bulk viscosity (defined in Ref. [23] for the on-shell case) reads in the relaxation time approximation (RTA) for the case of off-shell $\mathrm{DQPM}^{*}$ partons as:

$$
\begin{aligned}
\zeta\left(T, \mu_{q}\right)= & \frac{2}{9 T} d_{g} \int \frac{d^{3} p}{(2 \pi)^{3}} \int \frac{d \omega}{2 \pi} \omega \bar{\tau}_{g}\left(T, \mu_{q}\right) f_{g}(\omega / T) \rho_{g}(\omega, \mathbf{p}) \Theta\left(P^{2}\right) \frac{1}{\omega^{2}} F_{g}(\omega, \mathbf{p}) \\
& +\frac{2}{9 T} \frac{d_{q}}{6} \int \frac{d^{3} p}{(2 \pi)^{3}} \int \frac{d \omega}{2 \pi} \omega\left[\sum_{q}^{u, d, s} \bar{\tau}_{q}\left(T, \mu_{q}\right) f_{q}\left(\left(\omega-\mu_{q}\right) / T\right) \rho_{q}(\omega, \mathbf{p})\right. \\
& \left.+\sum_{\bar{q}}^{\bar{u}_{\bar{q}}, \bar{s}, \bar{s}} \bar{\tau}_{\bar{q}}\left(T, \mu_{q}\right) f_{\bar{q}}\left(\left(\omega+\mu_{q}\right) / T\right) \rho_{\bar{q}}(\omega, \mathbf{p})\right] \Theta\left(P^{2}\right) \frac{1}{\omega^{2}} F_{q}(\omega, \mathbf{p}),
\end{aligned}
$$

with

$$
F_{i}(\omega, \mathbf{p})=\left[\mathbf{p}^{2}-3 c_{s}^{2}\left(\omega^{2}-T^{2} \frac{d M_{i}^{2}}{d T^{2}}\right)\right]^{2}
$$


and essentially depends on the mass derivatives $\partial M_{i}^{2} / \partial T^{2}$, the temperature $T$, and the speed of sound squared $c_{s}^{2}$. All these quantities are accessible within the $\mathrm{DQPM}^{*}$ such that the results for the bulk viscosity again do not imply any new parameter.

The bulk viscosity (divided by the entropy density $s$ ) from the DQPM* is displayed in Fig. 5 (a) and shows a very different temperature dependence than $\eta / s$. Indeed, for high temperatures we find the limit $\zeta / s \rightarrow 0$. Moreover, the behavior around $T_{c}$ shows a peak in LCD as well as in the DQPM and DQPM* which is essentially due to the derivative $\partial M_{i}^{2} / \partial T^{2}$ in Eq. (4.4). Accordingly, the infrared enhancement in the $\mathrm{DQPM}^{*}$ masses is mandatory to achieve a maximum in the bulk viscosity $\zeta\left(T, \mu_{q}\right)$ to entropy ratio $\zeta / s$ close to $T_{c}$ in line with 1QCD. This enhancement close to $T_{c}$ is lower in the $\mathrm{DQPM}^{*}$ as in the DQPM probably due to a lower infrared enhancement in the coupling squared. Note, however, that such an enhancement does not show up in the NJL calculations for $\zeta / s$ from Ref. [10] (black solid line in (a)). The explicit dependencies of $\zeta / s$ on $T$ and $\mu_{q}$ from the DQPM* are shown in Fig. 5 (b).
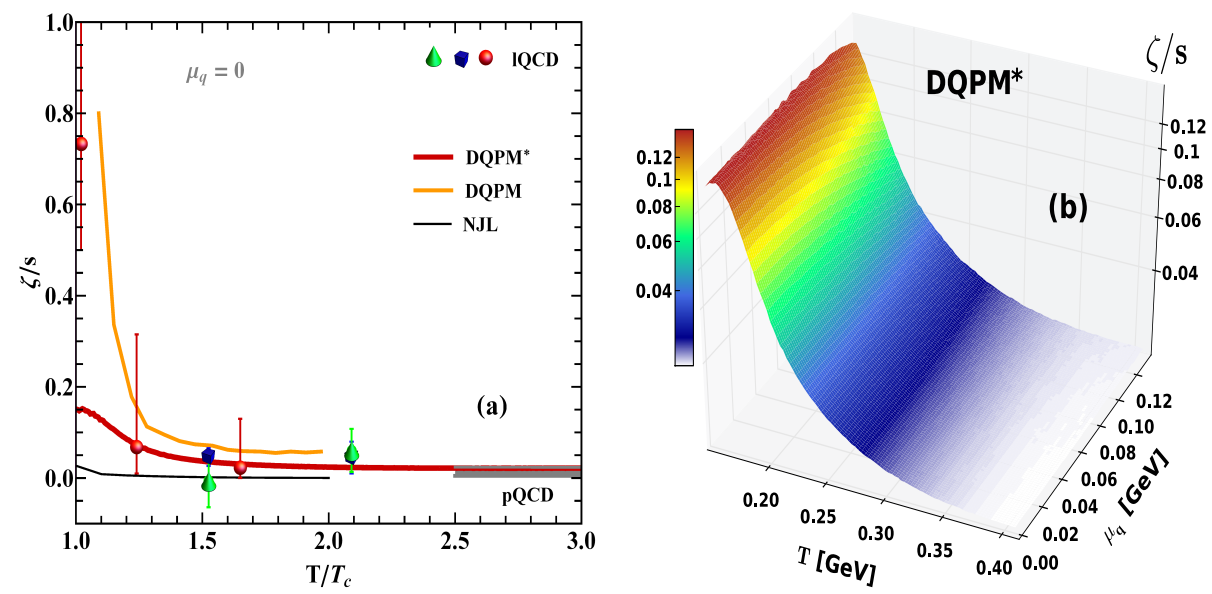

Figure 5: The bulk viscosity to entropy density ratio $\zeta / s$ from $D Q P M^{*}$ as a function of temperature $T$ for $\mu_{q}=0(a)$ and $\zeta / s$ given by the $D Q P M^{*}$ approach as a function of $T$ and $\mu_{q}(b)$. The orange solid line in (a) results from the standard DQPM where the parton masses and widths are independent of momenta [22]. The lattice QCD data points for pure SU (3) gauge theory are taken from Ref. [33] (red spheres), [30] (blue cubic) and from Ref. [29] (green pyramid). Finally, the black solid line in (a) refers to the calculation of $\zeta / s$ from the Nambu-Jona-Lasinio model for SU(3) from Ref. [10].

For $\mathrm{DQPM}^{*}$ results in case of the electric conductivity $\sigma_{e}\left(T, \mu_{q}\right)$ we refer the reader to Refs. $[17,31,32]$.

\section{Summary}

In this contribution we have reported about an extended dynamical quasiparticle model (DQPM*) incorporating momentum-dependent selfenergies in the parton propagators which are reflected in momentum-dependent masses and widths. Accordingly, the QGP effective degrees of freedom (above the critical temperature $T_{c}$ ) appear as interacting off-shell quasi-particles with masses and widths that depend on three-momentum $\mathbf{p}$, temperature $T$ and chemical potential $\mu_{q}$ as given in 
Eqs. (2.3). These expressions provide a proper high temperature limit (as in the HTL approximation) and approach the pQCD limit for large momenta $|\mathbf{p}|$. As in the standard DQPM the effective coupling is enhanced in the region close to $T_{c}$, which leads to an increase of the parton masses roughly below $1.2 T_{c}$ (cf. Fig. 1 (a)).

The extended dynamical quasiparticle model DQPM* reproduces quite well the 1 QCD results, i.e. the QGP equation of state, the baryon density $n_{B}$ and the quark susceptibility $\chi_{q}$ at finite temperature $T$ and quark chemical potential $\mu_{q}$ which had been a challenge for quasiparticle models so far [13] (see also Fig. 4b). A detailed comparison between the available lattice data and DQPM* results indicates a very good agreement for temperatures above $\sim 1.2 T_{c}$ in the pure partonic phase and therefore validates our description of the QGP thermodynamic properties. For temperatures in the vicinity of $T_{c}$ (and $\mu_{B}=400 \mathrm{MeV}$ ) we cannot expect our model to work so well since here hadronic degrees of freedom, which are discarded in the $\mathrm{DQPM}^{*}$, mix in a crossover phase. Furthermore, we have computed also the QGP shear viscosity $\eta$, the bulk viscosity $\zeta$, and the electric conductivity $\sigma_{e}$ at finite temperature and chemical potential in order to probe some transport properties of the medium. The results from the $\mathrm{DQPM}^{*}$ are found to be in line with the results from lattice QCD and are closer to the present 1QCD 'data' than the standard DQPM [17].

In view of our results on the description of the QGP thermodynamics and transport properties one can conclude that the $\mathrm{DQPM}^{*}$ provides a promising approach to study the QGP in equilibrium at finite temperature $T$ and chemical potential $\mu_{q}$. Moreover, we have demonstrated that one can simultaneously reproduce the 1 QCD pressure, the quark susceptibility and the QCD transport properties using a dynamical quasi-particle picture for the QGP effective degrees of freedom that allows for a transparent interpretation of the various results from lattice QCD. An implementation of the parton properties from the DQPM* into the PHSD transport approach $[34,35,36,37]$ is forseen in the near future.

\section{Acknowledgements}

The authors acknowledge valuable discussions with R. Marty, P. B. Gossiaux, J. Aichelin, O. Linnyk, P. Moreau, A. Palmese and E. Seifert. This work has been supported by the "HIC for FAIR" framework of the "LOEWE" program and BMBF. The computational resources have been provided by the LOEWE-CSC.

\section{References}

[1] S. Borsanyi, Z. Fodor, C. Hoelbling et al., PoS LATTICE2014 (2015) 224.

[2] S. Borsanyi, Z. Fodor, C. Hoelbling et al., Phys. Lett. B730 (2014) 99.

[3] M. I. Gorenstein, S.-N. Yang, Phys. Rev. D52 (1995) 5206.

[4] A. Peshier, B. Kämpfer, and G. Soff, Phys. Rev. C61 (2000) 045203.

[5] A. Peshier, B. Kämpfer, and G. Soff, Phys. Rev. D66 (2002) 094003.

[6] M. Bluhm, B. Kämpfer, and G. Soff, Phys. Lett. B620 (2005) 131.

[7] A. Peshier and W. Cassing, Phys. Rev. Lett. 94 (2005) 172301. 
[8] W. Cassing, Nucl. Phys. A795 (2007) 70.

[9] W. Cassing, Eur. Phys. J. ST 168 (2009) 3.

[10] R. Marty et al., Phys. Rev. C88 (2013) 045204.

[11] S. Borsanyi et al., JHEP 1208 (2012) 053.

[12] G. Endrodi, Z. Fodor, S. D. Katz, and K. Szabo, JHEP 04 (2011) 001.

[13] S. Plumari, W. M. Alberico, Wanda M., V. Greco, and C. Ratti, Phys. Rev. D84 (2011) 094004.

[14] B. Vanderheyden and G. Baym, J. Stat. Phys. 93 (1998) 843.

[15] C. Fischer, J. Phys. G32 (2006) R253-R291.

[16] C. Bonati et al., Phys. Rev. D90 (2014) 114025.

[17] H. Berrehrah, E. Bratkovskaya, T. Steinert, and W. Cassing, Int. J. Mod. Phys. E25 (2016) 1642003.

[18] H. Berrehrah, E. Bratkovskaya, W. Cassing, and T. Steinert, Phys. Rev. C93 (2016) 044914.

[19] J. P. Blaizot, E. Iancu, and A. Rebhan, Phys. Rev. D63 (2001) 065003.

[20] C. R. Allton et al., Phys. Rev. D68 (2003) 014507.

[21] C. R. Allton et al., Phys. Rev. D71 (2005) 054508.

[22] H. Berrehrah, E. Bratkovskaya, W. Cassing, and R. Marty, J. Phys. Conf. Ser. 612 (2015) 012050.

[23] P. Chakraborty and J. I. Kapusta, Phys. Rev. C83 (2011) 014906.

[24] V. Ozvenchuk et al., Phys. Rev. C87 (2013) 064903.

[25] S. Plumari, A. Puglisi, F. Scardina, and V. Greco, Phys. Rev. C86 (2012) 054902.

[26] N. Christiansen, M. Haas, J.M. Pawlowski, and N. Strodthoff, Phys. Rev. Lett. 115 (2015) 112002.

[27] L. P. Csernai, J. I. Kapusta, and L. D. McLerran, Phys. Rev. Lett. 97 (2006) 152303.

[28] P. Kovtun, D. T. Son, A. O. Starinets, Phys. Rev. Lett. 94 (2005) 111601.

[29] A. Nakamura and S. Sakai, Phys. Rev. Lett. 94 (2005) 072305.

[30] S. Sakai and A. Nakamura, PoS LAT2007 (2007) 221.

[31] W. Cassing, O. Linnyk, T. Steinert, and V. Ozvenchuk, Phys. Rev. Lett. 110 (2013) 182301.

[32] T. Steinert and W. Cassing, Phys. Rev. C89 (2014) 035203.

[33] H. B. Meyer, Phys. Rev. D76 (2007) 101701.

[34] W. Cassing and E. L. Bratkovskaya, Phys. Rev. C78 (2008) 034919; Nucl. Phys. A831 (2009) 215.

[35] E. L. Bratkovskaya, W. Cassing, V. P. Konchakovski, and O. Linnyk, Nucl. Phys. $\mathbf{A 8 5 6}$ (2011) 162.

[36] O. Linnyk, E. L. Bratkovskaya, and W. Cassing, Prog. Part. Nucl. Phys. 87 (2016) 50.

[37] W. Cassing, A. Palmese, P. Moreau, and E. L. Bratkovskaya, Phys. Rev. C93 (2016) 014902. 